\title{
Synthesis of ferrocenyl thioketones and their reactions with diphenyldiazomethane
}

\author{
Mlostoń, Grzegorz ; Hamera, Roza ; Heimgartner, Heinz
}

\begin{abstract}
A series of ferrocenyl ketones was obtained via Friedel-Crafts acylation with mixed anhydrides using ferrocene as a nucleophilic agent or ferrocene carboxylic acid as a precursor of the electrophilic species. The ketones obtained thereby undergo smooth thionation (THF, $65^{\circ} \mathrm{C}$ ) with Lawesson's reagent. The ferrocenyl thioketones react with diphenyldiazomethane via N2 elimination to afford the hitherto unknown ferrocenyl-substituted thiiranes.
\end{abstract}

DOI: https://doi.org/10.1080/10426507.2015.1071817

Posted at the Zurich Open Repository and Archive, University of Zurich

ZORA URL: https://doi.org/10.5167/uzh-116527

Journal Article

Accepted Version

Originally published at:

Mlostoń, Grzegorz; Hamera, Roza; Heimgartner, Heinz (2015). Synthesis of ferrocenyl thioketones and their reactions with diphenyldiazomethane. Phosphorus, Sulfur, and Silicon and the Related Elements, 190(12):2125-2133.

DOI: https://doi.org/10.1080/10426507.2015.1071817 


\title{
SYNTHESIS OF FERROCENYL THIOKETONES AND THEIR REACTIONS WITH DIPHENYLDIAZOMETHANE
}

\author{
Grzegorz MlostoD, ${ }^{1}$ * Ró| a Hamera, ${ }^{1}$ and Heinz Heimgartner ${ }^{2}$ \\ ${ }^{1}$ Department of Organic and Applied Chemistry, University of Aódz, Tamka 12, \\ PL 91-403 Aódz, Poland \\ ${ }^{2}$ Department of Chemistry, University of Zurich, Winterthurerstrasse 190, CH- \\ 8057 Zurich, Switzerland
}

Dedicated to Professor Emeritus Robert R. Holmes

\section{GRAPHICAL ABSTRACT}

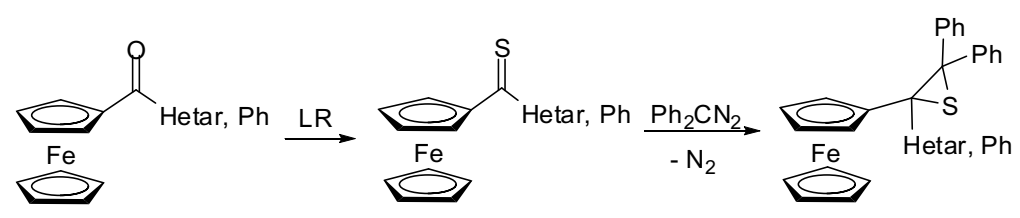

\begin{abstract}
A$ series of ferrocenyl ketones was obtained via Friedel-Crafts acylation with mixed anhydrides using ferrocene as a nucleophilic agent or ferrocene carboxylic acid as a precursor of the electrophilic species. The ketones obtained thereby undergo smooth thionation (THF, $\left.65{ }^{\circ} \mathrm{C}\right)$ with Lawesson's reagent. The ferrocenyl thioketones react with diphenyldiazomethane via $\mathrm{N}_{2}$ elimination to afford the hitherto unknown ferrocenyl-substituted thiiranes.
\end{abstract}

Keywords: ferrocene, thioketones, diazo compounds, thiiranes, thiophene derivatives, selenophene derivatives

Address correspondence to Grzegorz MlostoD, Department of Organic and Applied Chemistry, University of Aódz, Tamka 12, 91-403 Aódz, Poland. E-mail: gmloston@uni.lodz.pl

Part of the PhD thesis of R.H., University of Aódz 


\section{INTRODUCTION}

Aromatic thioketones are relatively stable compounds, which can be conveniently prepared from corresponding ketones by $\mathrm{O} / \mathrm{S}$ exchange using Lawessonïs reagent (LR). ${ }^{1}$ In the last three decades, aromatic thioketones and non-enolizable aliphatic thioketones have extensively been explored as versatile building blocks for the synthesis of more complex sulfur-containing compounds. Cycloaddition reactions leading to five- and six-membered S-heterocycles are of special importance, as thioketones are recognized as superdipolarophilic ${ }^{2}$ and superdienophilic agents. ${ }^{3}$ Moreover, thioketones are unique substrates for the preparation of differently substituted alkenes via the so-called 'two-fold extrusion reaction' with diazo compounds. ${ }^{4}$

Ferrocenyl-functionalized compounds are of great importance and found applications in materials chemistry, medicinal chemistry, polymer sciences, etc. ${ }^{5}$ Despite the growing interest in ferrocenyl-containing S-heterocycles, ${ }^{6}$ the ferrocenylsubstituted thioketones, which are attractive substrates for their synthesis, are only very little known. The synthesis of three representatives, i.e., ferrocenyl phenyl thioketone, ferrocenyl methyl thioketone, and tert-butyl ferrocenyl thioketones, was reported in 1978 without complete spectroscopic data. ${ }^{7}$ In addition, diferrocenyl thioketone was described as the product of thionation of diferrocenyl ketone. ${ }^{8}$

In our ongoing studies on the applications of thiocarbonyl compounds for the synthesis of S-heterocycles and related compounds, we reported the synthesis of diverse aryl hetaryl and dihetaryl thioketones, which are relatively stable and can be stored without decomposition. ${ }^{9}$ These results prompted us to elaborate a similar method for the preparation of ferrocenyl hetaryl ketones and subsequent thionation to give the corresponding thioketones.

\section{RESULTS AND DISCUSSION}

The best method for the preparation of ferrocenyl ketones 1 comprises FriedelCrafts acylation, and in the case of differocenyl ketone, the reported reaction was performed starting with ferrocene and ferrocenyl chloride in the presence of $\mathrm{AlCl}_{3}$ used in excess. ${ }^{8}$ However, in our hands, this protocol turned out to be inefficient, and in the 
best case, the desired ketone 1a, was obtained in only $25 \%$ yield. Therefore, we elaborated a new method based on the acylation via the in situ generated mixed anhydrides. ${ }^{10}$ In the case of 1a, ferrocenyl carboxylic acid was mixed with an equimolar amount of trifluoroacetic anhydride (TFAA) in the presence of trifluoroacetic acid (TFA) in $\mathrm{CH}_{2} \mathrm{Cl}_{2}$ solution at room temperature. Under these conditions, the added ferrocene underwent smooth acylation to give 1a in reproducible and satisfactory yield (52\%, Scheme 1). The same procedure was applied for the acylation of $N$-methylpyrrole and selenophene leading to the desired non-symmetric ketones $\mathbf{1 b}$ and $\mathbf{1 c}$ (84 and 88\% yield, resp.).

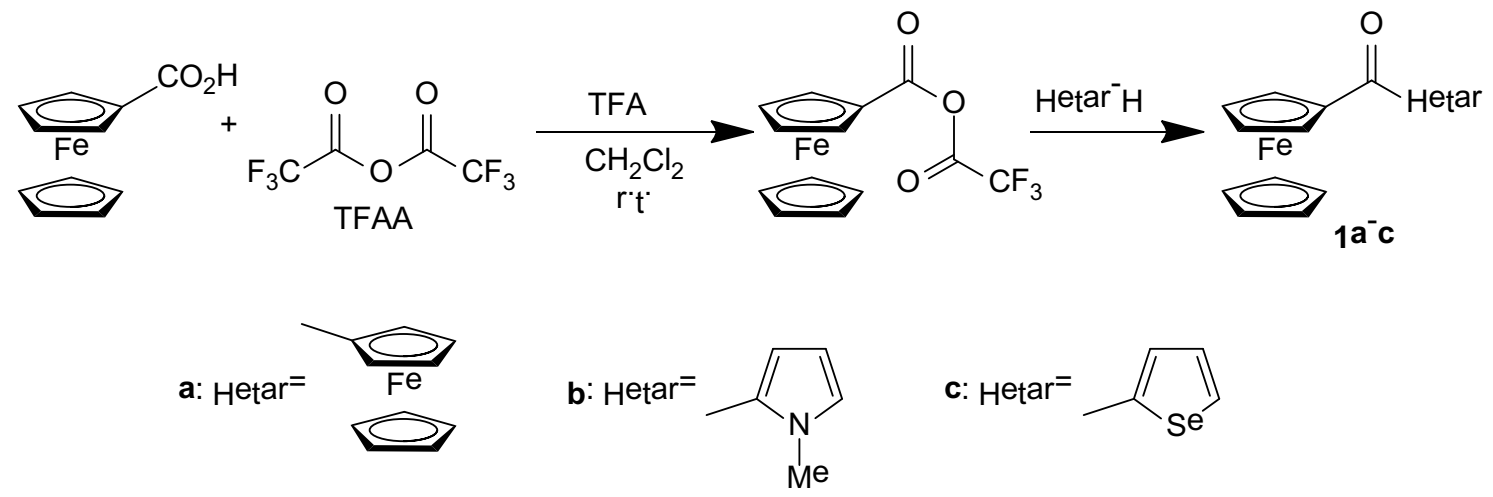

\section{Scheme 1}

A modified procedure was applied for the preparation of ferrocenyl phenyl ketone 1d, as the method presented above was unsuccessful. For that reason, the alternative mixed anhydride prepared from benzoic acid and TFAA in the presence of an equimolar amount of triflic acid (TFOH) in $\mathrm{CH}_{2} \mathrm{Cl}_{2}$ was prepared in situ at room temperature. Under these conditions, the added ferrocene underwent benzoylation to afford ketone 1d in 65\% yield (Scheme 2).

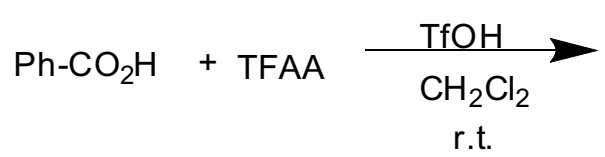<smiles>O=C(OC(=O)C(F)(F)F)c1ccccc1</smiles>

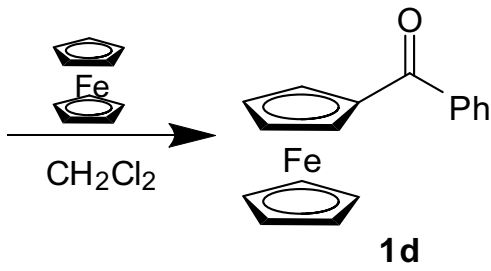

\section{Scheme 2}


The transformation of ketones $\mathbf{1}$ into the corresponding thioketones $\mathbf{2}$ was smoothly achieved by their treatment with LR in THF at $65^{\circ} \mathrm{C}$. The crude products were purified by column chromatography and isolated as green solids in $75-85 \%$ yield (Scheme 3).

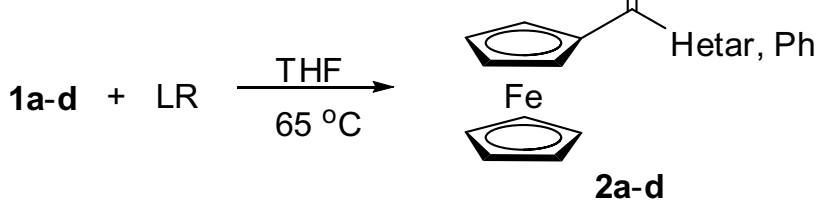

\section{Scheme 3}

Aromatic thioketones are known to react with diazomethane and its derivatives at low temperature yielding 2,5-dihydro-1,3,4-thiadiazoles as the products of [3+2] cycloadditions. ${ }^{11}$ Upon heating, these cycloadducts eliminate $\mathrm{N}_{2}$ and generate in situ the reactive thiocarbonyl ylides, which, depending on the type of substituents and the reaction conditions are converted into thiiranes, 1,4-dithianes, 1,3-dithiolanes, etc. The latter heterocycles were obtained as the sole products from the reaction of diazomethane with hetaryl thioketones. ${ }^{9}$ However, in the series of aryl selenophenyl thioketones, the intermediate thiocarbonyl ylides unexpectedly dimerize to give twelve-membered diselenaheterocycles via a postulated diradical intermediate. ${ }^{12}$

The test experiments with diazomethane and thioketone 2a were performed in THF solution at room temperature as well as at $-60{ }^{\circ} \mathrm{C}$. In both cases, immediate disappearance of the typical color of the thioketone was observed, but after a few minutes at room temperature, the solutions turned black and no defined products could be isolated. Similar reaction courses were observed in the attempted reactions of $\mathbf{2 a}$ with 2-diazopropane.

Finally, the reaction of $\mathbf{2 a}$ with the less reactive diphenyldiazomethane was performed in THF at room temperature. In that case, the TLC analysis evidenced the formation of a single product, which was identified as the symmetrical diferrocenylsubstituted thiirane $3 \mathbf{a}$ on the basis of spectroscopic data (Scheme 4). Thus, in the ${ }^{13} \mathrm{C}$ NMR spectrum, the diagnostic C-signals of the thiirane were found at 64.8 and 69.6 
ppm. Similarly, reactions of diphenyldiazomethane with $\mathbf{2 b - 2 d}$ occurred smoothly under the same conditions leading to non-symmetrical thiiranes $\mathbf{3 b}-\mathbf{3 d}$, respectively.

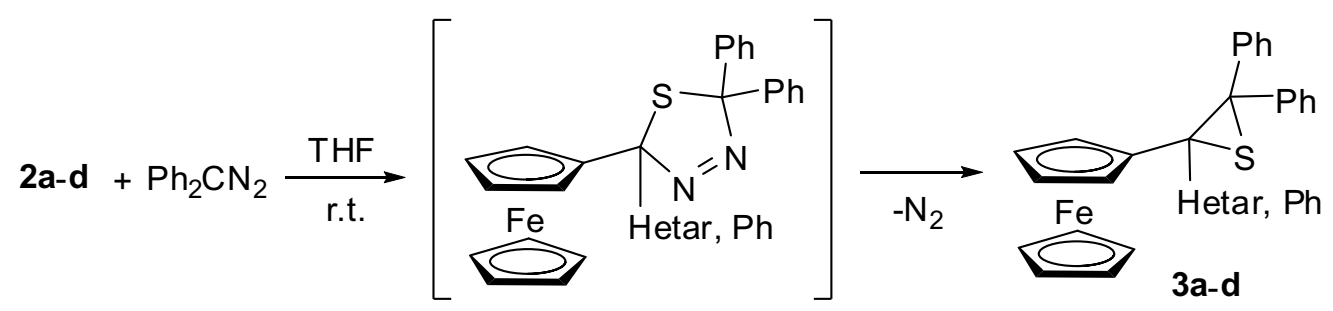

\section{Scheme 4}

It is worth of mentioning that the ferrocenyl-substituted thiiranes $\mathbf{3}$ are stable under standard conditions and can be used for further applications aimed at the preparation of more complex ferrocenyl containing products.

\section{CONCLUSIONS}

The method for the preparation of ferrocenyl ketones via Friedel-Crafts acylation with trifluoroacetyl-containing mixed anhydrides opens a straightforward access to this class of compounds. These ketones can be conveniently transformed into the corresponding aryl/hetaryl ferrocenyl thioketones. Their reactions with reactive diazo compounds like diazomethane or 2-diazopropane lead to decomposition. On the other hand, the less reactive diphenyldiazomethane reacts with ferrocenyl thioketones via spontaneous elimination of $\mathrm{N}_{2}$ to give ferrocenyl-substituted thiiranes as products of the 1,3-dipolar electrocyclization of the intermediate thiocarbonyl ylide.

\section{EXPERIMENTAL}

General Methods: All solvents were dried over appropriate drying agents and distilled before use. Reactions of the prepared ketones and thioketones were carried out under argon. Melting points were determined in a capillary using a Stewart® SMP30. The IR spectra (KBr pellets) were recorded on a Nexus FT-IR spectrometer. The ${ }^{1} \mathrm{H}$ and ${ }^{13} \mathrm{C}$ NMR spectra were measured on a Bruker Avance III $(600$ and $150 \mathrm{MHz}$, respectively) instrument, using $\mathrm{CHCl}_{3} / \mathrm{CDCl}_{3}$ signals as reference. ESI-MS were 
recorded on a Varian 500-MS IT Mass Spectrometer. The elemental analyses were recorded on a Vario Micro Cube. Flash chromatography was carried out using Silica gel 60 (Sigma-Aldrich, 230-400 mesh). The notation Fc in this study represents ferrocenyl.

Synthesis of ketones 1a-c (General procedure)

To a solution of ferrocenecarboxylic acid $(2.17 \mathrm{mmol}, 0.5 \mathrm{~g})$ in dry $\mathrm{CH}_{2} \mathrm{Cl}_{2}(10$ $\mathrm{ml}$ ) was added trifluoroacetic anhydride (TFAA, $2.17 \mathrm{mmol}, 0.3 \mathrm{ml}$ ). After stirring the mixture for ca. $2 \mathrm{~min}$ at room temperature, trifluoroacetic acid (TFA, $2.17 \mathrm{mmol}, 0.16$ $\mathrm{ml})$ and the corresponding hetarene $(2.17 \mathrm{mmol})$ were added. The progress of the reaction was monitored by thin layer chromatography (TLC). After completion of the reaction, water $(15 \mathrm{ml})$ was added. The organic layer was separated and the aqueous layer was extracted with $\mathrm{CH}_{2} \mathrm{Cl}_{2}$. The combined organic extracts were dried $\left(\mathrm{MgSO}_{4}\right)$ and the solvent evaporated. Crude products were purified by flash chromatography $\left(\mathrm{CH}_{2} \mathrm{Cl}_{2} / \mathrm{CH}_{3} \mathrm{OH} 99: 1\right)$.

\section{Diferrocenyl ketone (1a)}

Yield: 449 mg (52\%). Orange solid. M.p.: 203.1-204.3 ${ }^{\circ} \mathrm{C} .{ }^{1} \mathrm{H}$ NMR: ' 4.21 (s, $10 \mathrm{H}, \mathrm{CH}-\mathrm{Fc}), 4.53\left(\mathrm{t}, J_{\mathrm{H}, \mathrm{H}}=1.8 \mathrm{~Hz}, 4 \mathrm{H}, \mathrm{CH}-\mathrm{Fc}\right), 5.00$ (t, $\left.J_{\mathrm{H}, \mathrm{H}}=1.8 \mathrm{~Hz}, 4 \mathrm{H}, \mathrm{CH}-\mathrm{Fc}\right)$ ppm. ${ }^{13} \mathrm{C}$ NMR: ' 70.0, 70.6, 71.4 (18C, CH-Fc), 80.5 (2C, C-Fc), $199.2(1 \mathrm{C}, \mathrm{C}=\mathrm{O})$ ppm. IR: Å3123 (m), 3105 (m), 3096 (m), 3089 (m), 1772 (w), 1608 (vs, C=O), 1541 (m), 1465 (vs), 1413 (m), 1395 (m), 1377 (s), 1355 (m), 1293 (vs), 1202 (m), 1106 (s), 1064 (s), 1054 (m), 1027 (m), 1009 (m), 904 (m), 836 (s), 819 (s), 807 (vs), 771 (m), $580(\mathrm{~m}), 510(\mathrm{~m}), 493$ (vs), 478 (vs) $\mathrm{cm}^{-1}$. ESI-MS $400\left(8,[\mathrm{M}+2]^{+}\right), 421(100$, $\left.[\mathrm{M}+\mathrm{Na}]^{+}\right), 422\left(24,[\mathrm{M}+\mathrm{Na}+1]^{+}\right)$.

\section{Ferrocenyl $N$-methylpyrrol-2-yl ketone (1b)}

Yield: 534 mg (84\%). Red solid. M.p.: 113.9-115.4 ${ }^{\circ} \mathrm{C} .{ }^{1} \mathrm{H}$ NMR: ' 3.97 (s, 3H, $\left.\mathrm{CH}_{3}\right), 4.22(\mathrm{~s}, 5 \mathrm{H}, \mathrm{CH}-\mathrm{Fc}), 4.50\left(\mathrm{t}, J_{\mathrm{H}, \mathrm{H}}=1.8 \mathrm{~Hz}, 2 \mathrm{H}, \mathrm{CH}-\mathrm{Fc}\right), 4.95\left(\mathrm{t}, J_{\mathrm{H}, \mathrm{H}}=1.8 \mathrm{~Hz}\right.$, $2 \mathrm{H}, \mathrm{CH}-\mathrm{Fc}), 6.16\left(\mathrm{dd}, J_{\mathrm{H}, \mathrm{H}}=3.6,2.4 \mathrm{~Hz}, 1 \mathrm{H}, \mathrm{CH}_{\text {arom }}\right), 6.84\left(\mathrm{dd}, J_{\mathrm{H}, \mathrm{H}}=2.4,1.2 \mathrm{~Hz}, 1 \mathrm{H}\right.$, $\mathrm{CH}_{\text {arom. }}$ ), $7.14\left(\mathrm{dd}, J_{\mathrm{H}, \mathrm{H}}=3.6,1.2 \mathrm{~Hz}, 1 \mathrm{H}, \mathrm{CH}_{\text {arom. }}\right)$ ppm. ${ }^{13} \mathrm{C} \mathrm{NMR}$ : ' $37.2\left(1 \mathrm{C}, \mathrm{CH}_{3}\right)$, 70.0, 71.0, 71.3 (9C, CH-Fc), 80.8 (1C, C-Fc), 107.6, 118.8, 129.8 (3C, $\left.\mathrm{CH}_{\text {arom. }}\right), 131.3$ (1C, $\mathrm{C}_{\text {arom. }}$ ), 187.9 (1C, C=O) ppm. 70.4, 71.0, 72.2 (9 CH-Fc), 78.6 (C-Fc), 130.4, 133.4, 137.8 (3 $\left.\mathrm{CH}_{\text {arom. }}\right), 151.4\left(\mathrm{C}_{\text {arom. }}\right), 190.5$ (C=O) ppm. IR: $\AA 3118(\mathrm{w}), 3105$ (m), 3082 (w), 2927 (w), 1600 (vs, C=O), 1524 (m), 1444 (vs), 1405 (vs), 1379 (s), 1320 
(m), 1261 (s), 1206 (w), 1174 (w), 1066 (m), 1047 (m), 1021 (m), 940 (w), 844 (m), 836 (m), 823 (s), 745 (vs), 609 (m), 565 (m), 503 (s), 482 (s) cm ${ }^{-1}$. ESI-MS $293\left(20,[\mathrm{M}]^{+}\right.$), $294\left(100,[\mathrm{M}+1]^{+}\right)$. Anal. calcd. for $\mathrm{C}_{16} \mathrm{H}_{15} \mathrm{FeNO}$ (293.14): C 65.56, H 5.16, N 4.78; found: C 65.37, H 5.03, N 4.63.

\section{Ferrocenyl selenophen-2-yl ketone (1c)}

Yield: 655 mg (88\%). Red solid. M.p.: 113.5-115.0 ${ }^{\circ} \mathrm{C} .{ }^{1} \mathrm{H}$ NMR:' 4.24 (s, 5H, $\mathrm{CH}-\mathrm{Fc}), 4.60\left(\mathrm{t}, J_{\mathrm{H}, \mathrm{H}}=1.8 \mathrm{~Hz}, 2 \mathrm{H}, \mathrm{CH}-\mathrm{Fc}\right), 5.02\left(\mathrm{t}, J_{\mathrm{H}, \mathrm{H}}=1.8 \mathrm{~Hz}, 2 \mathrm{H}, \mathrm{CH}-\mathrm{Fc}\right), 7.44$ (dd, $\left.J_{\mathrm{H}, \mathrm{H}}=5.4,3.6 \mathrm{~Hz}, 1 \mathrm{H}, \mathrm{CH}_{\text {arom. }}\right), 8.15\left(\mathrm{dd}, J_{\mathrm{H}, \mathrm{H}}=3.6,1.2 \mathrm{~Hz}, 1 \mathrm{H}, \mathrm{CH}_{\text {arom. }}\right), 8.35\left(\mathrm{dd}, J_{\mathrm{H}, \mathrm{H}}\right.$

$\left.=5.4,1.2 \mathrm{~Hz}, 1 \mathrm{H}, \mathrm{CH}_{\text {arom. }}\right)$ ppm. ${ }^{13} \mathrm{C} \mathrm{NMR}$ : ' 70.4, 71.0, 72.2 (9C, CH-Fc), 78.6 (1C, CFc), 130.4, 133.4, 137.8 (3C, $\left.\mathrm{CH}_{\text {arom. }}\right), 151.4$ (1C, $\left.\mathrm{C}_{\text {arom. }}\right), 190.5$ (1C, C=O) ppm. IR: $\AA$ 3096 (m), 3078 (m), 1767 (w), 1710 (w), 1594 (vs, C=O), 1557 (m), 1524 (s), 1443 (s), 1422 (vs), 1376 (s), 1290 (vs), 1152 (s), 1041 (vs), 823 (s), 769 (s), 747 (s), 710 (vs), $589(\mathrm{w}), 520(\mathrm{~m}), 498(\mathrm{~s}), 485(\mathrm{~s}) \mathrm{cm}^{-1}$. ESI-MS $343\left(45,[\mathrm{M}]^{+}\right), 344\left(73,[\mathrm{M}+1]^{+}\right), 345$ $\left(100,[\mathrm{M}+2]^{+}\right), 365\left(58,[\mathrm{M}+\mathrm{Na}-1]^{+}\right)$. Anal. calcd. for $\mathrm{C}_{15} \mathrm{H}_{12} \mathrm{FeOSe}(343.06)$ : C 52.52, H 3.53; found: C 52.22, H 3.51.

\section{Synthesis of ketone 1d}

A solution of benzoic acid (2.69 mmol, $0.33 \mathrm{~g})$ and TFAA $(2.69 \mathrm{mmol}, 0.37 \mathrm{ml})$ in dry $\mathrm{CH}_{2} \mathrm{Cl}_{2}(10 \mathrm{ml})$ was stirred ca. $2 \mathrm{~min}$ at room temperature. Then, triflic acid (TfOH, $2.69 \mathrm{mmol}, 0.16 \mathrm{ml})$ and ferrocene $(2.69 \mathrm{mmol}, 0.5 \mathrm{~g})$ were added. The progress of the reaction was monitored by TLC. The subsequent work-up procedure was analogous to the one described for ketones $\mathbf{1 a}-\mathbf{1 c}$.

\section{Ferrocenyl phenyl ketone (1d)}

Yield: 507 mg (65\%). Red solid. M.p.: 105.0-106.5 ${ }^{\circ} \mathrm{C} .{ }^{1} \mathrm{H}$ NMR: ' 4.20 (s, 5H, $\mathrm{CH}-\mathrm{Fc}), 4.58\left(\mathrm{t}, J_{\mathrm{H}, \mathrm{H}}=1.8 \mathrm{~Hz}, 2 \mathrm{H}, \mathrm{CH}-\mathrm{Fc}\right), 4.90\left(\mathrm{t}, J_{\mathrm{H}, \mathrm{H}}=1.8 \mathrm{~Hz}, 2 \mathrm{H}, \mathrm{CH}-\mathrm{Fc}\right), 7.46(\mathrm{t}$, $\left.J_{\mathrm{H}, \mathrm{H}}=7.7 \mathrm{~Hz}, 2 \mathrm{H}, \mathrm{CH}_{\text {arom. }}\right), 7.54\left(\mathrm{t}, J_{\mathrm{H}, \mathrm{H}}=7.3 \mathrm{~Hz}, 1 \mathrm{H}, \mathrm{CH}_{\text {arom. }}\right), 7.89\left(\mathrm{~d}, J_{\mathrm{H}, \mathrm{H}}=7.3 \mathrm{~Hz}\right.$, $2 \mathrm{H}, \mathrm{CH}_{\text {arom. }}$ ) ppm. ${ }^{13} \mathrm{C}$ NMR: ' 70.2, 71.5, 72.5 (9C, CH-Fc), 78.2 (1C, C-Fc), 128.0, 128.2, 131.4 (5C, $\left.\mathrm{CH}_{\text {arom. }}\right), 139.9$ (1C, $\left.\mathrm{C}_{\text {arom. }}\right), 199.0(1 \mathrm{C}, \mathrm{C}=\mathrm{O})$ ppm. IR: $\AA 3113$ (w), 3091 (w), 3065 (w), 1626 (vs, C=O), 1597 (s), 1577 (s), 1450 (s), 1440 (s), 1375 (s), 1289 (vs), 1166 (m), 1057 (m), 1025 (s), 1003 (m), 952 (m), 826 (s), 724 (vs), 696 (s), $676(\mathrm{~m}), 564(\mathrm{~m}) \mathrm{cm}^{-1}$. ESI-MS $290\left(31,[\mathrm{M}]^{+}\right), 291\left(100,[\mathrm{M}+1]^{+}\right), 313\left(54,[\mathrm{M}+\mathrm{Na}]^{+}\right)$. Anal. calcd. for $\mathrm{C}_{17} \mathrm{H}_{14} \mathrm{FeO}$ (290.14): C 70.37, H 4.86; found: C 70.41, H 4.83. 


\section{Synthesis of thioketones $2 \mathbf{a}-\mathbf{d}$ (General procedure)}

The solution of the corresponding ketone 1 ( $1 \mathrm{mmol})$ in THF or benzene $(5 \mathrm{ml})$ was heated to $65{ }^{\circ} \mathrm{C}$ and next Lawesson's reagent (LR, $0.6 \mathrm{mmol}, 0.24 \mathrm{~g}$ ) was added. After complete reaction (TLC), the mixture was concentrated and the crude product was purified by flash chromatography (hexane/ $\mathrm{CH}_{2} \mathrm{Cl}_{2} 7: 3$ ).

\section{Diferrocenyl thioketone (2a)}

Yield: $311 \mathrm{mg}$ (75\%). Violet solid. M.p.: $>135{ }^{\circ} \mathrm{C}$ (decomposition) [lit. ${ }^{8}$, m.p. $135{ }^{\circ} \mathrm{C}$ (decomp.)]. ${ }^{1} \mathrm{H}$ NMR: ' 4.20 (s, 10H, CH-Fc), 4.68 (s, 4H, CH-Fc), 5.22 (s, 4H, CH-Fc) ppm. ${ }^{13} \mathrm{C}$ NMR: ' 71.3, 72.5, 72.6 (18C, CH-Fc), 90.4 (2C, C-Fc), 237.1 (1C, $\mathrm{C}=\mathrm{S})$ ppm. IR: A3 3120 (w), 3096 (m), 3084 (m), 2958 (w), 2922 (m), $2851(\mathrm{w}), 1677$ (m), 1654 (m), 1623 (m), 1594 (m), 1443 (vs), 1430 (s), 1400 (m), 1378 (m), 1352 (m), 1334 (m), 1286 (s), 1249 (vs), 1206 (m), 1190 (m), 1105 (m), 1073 (s), 1045 (s), 1004 (s), 945 (m), 887 (m), 873 (m), 847 (m), 830 (s), 817 (vs), 767 (m), 696 (m), 546 (m), 516 (s), 484 (vs), $474(\mathrm{vs}), 444(\mathrm{~m}) \mathrm{cm}^{-1}$. ESI-MS $414\left(4,[\mathrm{M}]^{+}\right), 415\left(100,[\mathrm{M}+1]^{+}\right)$, $416\left(26,[\mathrm{M}+2]^{+}\right)$.

\section{Ferrocenyl $N$-methylpyrrol-2-yl thioketone (2b)}

Yield: $263 \mathrm{mg}$ (85\%). Violet solid. M.p.: 102.0-102.8 ${ }^{\circ} \mathrm{C} .{ }^{1} \mathrm{H}$ NMR: ' 3.97 (s, $\left.3 \mathrm{H}, \mathrm{CH}_{3}\right), 4.18(\mathrm{~s}, 5 \mathrm{H}, \mathrm{CH}-\mathrm{Fc}), 4.68\left(\mathrm{t}, J_{\mathrm{H}, \mathrm{H}}=1.8 \mathrm{~Hz}, 2 \mathrm{H}, \mathrm{CH}-\mathrm{Fc}\right), 5.11\left(\mathrm{t}, J_{\mathrm{H}, \mathrm{H}}=1.8\right.$ $\mathrm{Hz}, 2 \mathrm{H}, \mathrm{CH}-\mathrm{Fc}), 6.14$ (dd, $J_{\mathrm{H}, \mathrm{H}}=4.2,2.4 \mathrm{~Hz}, 1 \mathrm{C}, \mathrm{CH}_{\mathrm{arom}}$ ), 6.85 (dd, $J_{\mathrm{H}, \mathrm{H}}=4.2,1.8 \mathrm{~Hz}$, $1 \mathrm{C}, \mathrm{CH}_{\text {arom. }}$ ), $7.72\left(\mathrm{dd}, J_{\mathrm{H}, \mathrm{H}}=2.4,1.8 \mathrm{~Hz}, 1 \mathrm{C}, \mathrm{CH}_{\text {arom. }}\right.$ ) ppm. ${ }^{13} \mathrm{C} \mathrm{NMR}$ : $37.4\left(\mathrm{CH}_{3}\right)$, 71.6, 72.8, 72.9 (9C, CH-Fc), 91.1 (1C, C-Fc), 107.6, 117.1, 132.9 (3C, $\mathrm{CH}_{\text {arom. }}$ ), 142.4 (1C, $\left.\mathrm{C}_{\text {arom. }}\right), 218.0(1 \mathrm{C}, \mathrm{C}=\mathrm{S})$ ppm. IR: $\AA 3105$ (m), 2948 (w), $1636(\mathrm{w}), 1518(\mathrm{~m}), 1454$ (s), 1432 (vs), 1401 (vs), 1271 (vs), 1242 (m), 1188 (m), 1068 (s), 1048 (s), 1020 (s), 1002 (m), 830 (m), 821 (m), 784 (s), 742 (vs), 702 (m), 604 (m), 493 (s) cm ${ }^{-1}$. ESI-MS $309\left(54,[\mathrm{M}]^{+}\right), 310\left(100,[\mathrm{M}+1]^{+}\right)$. Anal. calcd. for $\mathrm{C}_{16} \mathrm{H}_{15} \mathrm{FeNS}(309.21)$ : C 62.15, H 4.89, N 4.53, S 10.37; found: C 62.36, H 4.94, N 4.53, S 10.75.

\section{Ferrocenyl selenophen-2-yl thioketone (2c)}

Yield: $298 \mathrm{mg}$ (83\%). Green solid. M.p.: 83.5-85.0 ${ }^{\circ} \mathrm{C} .{ }^{1} \mathrm{H}$ NMR: ' 4.22 (s, 5H, $\mathrm{CH}-\mathrm{Fc}$ ), 4.78 (s, 2H, CH-Fc), 5.15 (s, 2H, CH-Fc), 7.45 (brs/m, 1H, $\mathrm{CH}_{\text {arom. }}$ ), 7.94 (brs $/ \mathrm{m}, 1 \mathrm{H}, \mathrm{CH}_{\text {arom. }}$ ), 8.35 (brs/m, 1H, $\mathrm{CH}_{\text {arom. }}$ ) ppm. ${ }^{13} \mathrm{C} \mathrm{NMR}:{ }^{\prime} 72.2,73.0,73.8$ (9C, 
CH-Fc), 88.5 (1C, C-Fc), 129.3, $131.2,141.4$ (3C, $\left.\mathrm{CH}_{\text {arom. }}\right), 162.0$ (1C, $\mathrm{C}_{\text {arom. }}$ ), 224.9 (1C, C=S) ppm. IR: Å3083 (m), 1503 (m), 1432 (vs), 1410 (vs), 1379 (s), 1332 (vs), 1285 (v), 1274 (s), 1228 (vs), 1214 (s), 1171 (m), 1107 (m), 1058 (s), 1042 (s), 1001 (m), $977(\mathrm{~m}), 860$ (s), 830 (s), 808 (s), 716 (vs), 681 (m), 641 (m), 588 (m), 506 (s), 483 (s) $\mathrm{cm}^{-1}$. ESI-MS $359\left(48,[\mathrm{M}]^{+}\right), 361\left(100,[\mathrm{M}+2]^{+}\right)$. Anal. calcd. for $\mathrm{C}_{15} \mathrm{H}_{12} \mathrm{FeSSe}$ (359.13): C 50.17, H 3.37, S 8.93; found: C 50.25, H 3.42, S 8.97.

\section{Ferrocenyl phenyl thioketone (2d)}

Yield: $254 \mathrm{mg}$ (83\%). Violet solid. M.p.: 71.2-72.5 ${ }^{\circ} \mathrm{C} .{ }^{1} \mathrm{H}$ NMR: ' 4.17 (s, 5H, $\mathrm{CH}-\mathrm{Fc}$ ), 4.82 (s, 2H, CH-Fc), 5.05 (s, 2H, CH-Fc), 7.37 (brs/m, 2H, $\mathrm{CH}_{\text {arom.) }}$ ), 7.47 (brs/m, 1H, $\mathrm{CH}_{\text {arom. }}$ ), 7.67 (d, $J_{\mathrm{H}, \mathrm{H}}=6.0 \mathrm{~Hz}, 2 \mathrm{H}, \mathrm{CH}_{\text {arom. }}$ ) ppm. ${ }^{13} \mathrm{C} \mathrm{NMR}$ : ' 72.1, 72.6, 74.8 (9C, CH-Fc), 89.4 (1C, C-Fc), 126.9, 127.6, 130.1 (5C, $\mathrm{CH}_{\text {arom. }}$ ), 149.0 (1C, $\mathrm{C}_{\text {arom. }}$ ), 238.7 (1C, C=S) ppm. IR: $\AA 3108$ (w), 3095 (w), 3073 (w), 3058 (w), 2925 (w), 2852 (w), 1592 (m), 1444 (s), 1431 (vs), 1398 (m), 1374 (s), 1294 (s), 1280 (vs), 1242 (vs), 1175 (m), 1108 (m), 1087 (m), 1065 (s), 1007 (vs), 932 (m), 827 (vs), 768 (vs), 701 (vs), 684 (s), 635 (m), 506 (vs), 483 (vs) cm ${ }^{-1}$. ESI-MS 230 (24, [M-Ph+1] $\left.]^{+}\right), 307$ (100, $\left.[\mathrm{M}+1]^{+}\right), 308\left(25,[\mathrm{M}+2]^{+}\right)$. Anal. calcd. for $\mathrm{C}_{17} \mathrm{H}_{14} \mathrm{FeS}(306.20)$ : C 66.68, H 4.61, S 10.47; found: C 66.82, H 4.73, S 10.42.

\section{Synthesis of thiiranes 3a-d (General procedure)}

To a solution of the appropriate thioketone $2(1 \mathrm{mmol})$ in dry THF $(4 \mathrm{ml})$ was added a solution of diphenyldiazomethane $(1 \mathrm{mmol}, 0.19 \mathrm{~g})$ in dry THF $(2 \mathrm{ml})$. After stirring overnight, the reaction was completed (according to TLC). The solvent was evaporated and the product was purified by flash chromatography (hexane/ $\mathrm{CH}_{2} \mathrm{Cl}_{2} 7: 3$ ).

\section{3,3-Diferrocenyl-2,2-diphenylthiirane (3a)}

Yield: $320 \mathrm{mg}$ (55\%). Orange solid. M.p.: $>183{ }^{\circ} \mathrm{C}$ (decomposition). ${ }^{1} \mathrm{H}$ NMR: 3.95-3.97 (m, 2H, CH-Fc), 4.04 (s, 10H, CH-Fc), 4.09-4.10 (m, 2H, CH-Fc), 4.114.12 (m, 2H, CH-Fc), 4.13-4.14 (m, 2H, CH-Fc), 7.09-7.13 (m, 2H, $\mathrm{CH}_{\text {arom. }}$ ), 7.15-7.19 (m, 4H, $\mathrm{CH}_{\text {arom. }}$ ), 7.42 (brs, 4H, $\mathrm{CH}_{\text {arom. }}$ ) ppm. ${ }^{13} \mathrm{C}$ NMR:' 59.1, 72.3 (2C, Cq), 66.4, 67.4, 69.5, 70.7, 70.8 (18C, CH-Fc), 92.6 (2C, C-Fc), 126.5, 127.6, 130.1 (10C, $\left.\mathrm{CH}_{\text {arom. }}\right), 143.9$ (2C, $\left.\mathrm{C}_{\text {arom. }}\right)$ ppm. IR: $\AA 3091$ (m), 3082 (m), 3053 (w), 3026 (w), 1595 (m), 1487 (m), 1444 (m), 1412 (m), 1273 (m), 1199 (m), 1158 (m), 1106 (m), 1063 (m), 1025 (m), 1000 (m), 817 (s), 807 (s), 780 (m), 745 (m), 704 (vs), 689 (s), 633 (m), 605 
(m), $571(\mathrm{~m}), 529(\mathrm{~m}), 490(\mathrm{vs}) \mathrm{cm}^{-1}$. ESI-MS $548\left(100,[\mathrm{M}-\mathrm{S}]^{+}\right), 549\left(49,[\mathrm{M}-\mathrm{S}+1]^{+}\right)$, $580\left(79,[\mathrm{M}]^{+}\right), 581\left(40,[\mathrm{M}+1]^{+}\right)$. Anal. calcd. for $\mathrm{C}_{34} \mathrm{H}_{28} \mathrm{Fe}_{2} \mathrm{~S}(580.34): \mathrm{C} 70.37, \mathrm{H}$ 4.86, S 5.53; found: C 70.62, H 4.63, S 5.59.

\section{3-Ferrocenyl-2,2-diphenyl-3-( $N$-methylpyrrol-2-yl)thiirane (3b)}

Yield: $252 \mathrm{mg}$ (53\%). Yellow solid. M.p.: $>73{ }^{\circ} \mathrm{C}$ (decomposition). ${ }^{1} \mathrm{H}$ NMR:

3.11 (s, 3H, $\mathrm{CH}_{3}$ ), 3.40-3.41 (m, 1H, CH-Fc), 3.81-3.82 (m, 1H, CH-Fc), 3.84-3.85 (m, $1 \mathrm{H}, \mathrm{CH}-\mathrm{Fc}), 4.00-4.01(\mathrm{~m}, 1 \mathrm{H}, \mathrm{CH}-\mathrm{Fc}), 4.13(\mathrm{~s}, 5 \mathrm{H}, \mathrm{CH}-\mathrm{Fc}), 6.11\left(\mathrm{dd}, J_{\mathrm{H}, \mathrm{H}}=3.6,3.0\right.$ $\left.\mathrm{Hz}, 1 \mathrm{H}, \mathrm{CH}_{\text {arom. }}\right), 6.25\left(\mathrm{t}, J_{\mathrm{H}, \mathrm{H}}=1.8 \mathrm{~Hz}, 1 \mathrm{H}, \mathrm{CH}_{\text {arom. }}\right), 6.60\left(\mathrm{dd}, J_{\mathrm{H}, \mathrm{H}}=3.6,2.4 \mathrm{~Hz}, 1 \mathrm{H}\right.$, $\mathrm{CH}_{\text {arom. }}$ ), 6.96-7.0 (m, 2H, $\mathrm{CH}_{\text {arom. }}$ ), 7.02-7.08 (m, 3H, $\left.\mathrm{CH}_{\text {arom. }}\right), 7.21-7.26$ (m, 5H, $\mathrm{CH}_{\text {arom. }}$ ) ppm. ${ }^{13} \mathrm{C}$ NMR: ' $34.4\left(1 \mathrm{C}, \mathrm{CH}_{3}\right), 57.3,69.0$ (2C, $\left.\mathrm{Cq}\right), 67.3,67.4,69.4,69.9$, 70.8 (9C, CH-Fc), 92.1 (1C, C-Fc), 106.7, 114.3, 120.9, 126.6, 126.8, 127.0, 129.0, 132.3 (13C, $\left.\mathrm{CH}_{\text {arom. }}\right), 131.6,139.6,140.3$ (3C, $\mathrm{C}_{\text {arom. }}$ ) ppm. IR: $\AA 3085$ (w), 3057 (w), 2953 (w), 2926 (w), 1491 (m), 1444 (m), 1307 (m), 1106 (m), 1078 (w), 1055 (w), 1030 (w), 1002 (m), 818 (m), 783 (m), 753 (m), 736 (m), 699 (s), 609 (w), 498 (m) cm c $^{-1}$ ESI-MS $443\left(28,[\mathrm{M}-\mathrm{S}]^{+}\right), 475\left(100,[\mathrm{M}]^{+}\right), 476\left(40,[\mathrm{M}+1]^{+}\right)$. Anal. calcd. for $\mathrm{C}_{29} \mathrm{H}_{25} \mathrm{FeNS}$ (475.43): C 73.26, H 5.30, N 2.95, S 6.74; found: C 73.32 H 5.41, N 3.04, S 6.69

\section{3-Ferrocenyl-2,2-diphenyl-3-(selenophen-2-yl)thiirane (3c)}

Yield: $298 \mathrm{mg}$ (57\%). Yellow solid. M.p.: 125.0-126.7 ${ }^{\circ} \mathrm{C} .{ }^{1} \mathrm{H}$ NMR: ' 3.66 (s, 1H, CH-Fc), 3.87 (s, 1H, CH-Fc), 4.07 (s, 5H, CH-Fc), 4.09 (s, 1H, CH-Fc), 4.39 (s, $1 \mathrm{H}, \mathrm{CH}-\mathrm{Fc}$ ), 6.98-7.08 (m, 4H, $\left.\mathrm{CH}_{\text {arom. }}\right), 7.13$ (brs, 3H, $\left.\mathrm{CH}_{\text {arom. }}\right), 7.19-7.27$ (m, 3H, $\left.\mathrm{CH}_{\text {arom. }}\right), 7.30\left(\mathrm{~d}, J_{\mathrm{H}, \mathrm{H}}=6.6 \mathrm{~Hz}, 2 \mathrm{H}, \mathrm{CH}_{\text {arom. }}\right), 7.76\left(\mathrm{~d}, J_{\mathrm{H}, \mathrm{H}}=4.8 \mathrm{~Hz}, 1 \mathrm{H}, \mathrm{CH}_{\text {arom. }}\right)$ ppm. ${ }^{13}$ C NMR: ' 60.7, 72.2 (2C, Cq), 67.6, 68.2, 69.2, 70.5, 71.1 (9C, CH-Fc), 90.2 (1C, CFc), 126.5, 126.7, 127.2, 128.2, 129.4, 130.4, 131.1, 131.2 (13C, $\left.\mathrm{CH}_{\text {arom. }}\right), 140.4,141.2$, 151.9 (3C, C arom.) ppm. IR: Å3082 (m), 3055 (m), 3026 (m), 2922 (m), 2850 (m), 1598 (m), 1490 (m), 1444 (m), 1227 (m), 1106 (m), 1079 (m), 1032 (m), 1001 (m), 818 (m), $777(\mathrm{~m}), 750(\mathrm{~m}), 696(\mathrm{~s}), 658(\mathrm{~m}), 604(\mathrm{~m}), 491(\mathrm{~m}) \mathrm{cm}^{-1}$. ESI-MS $524\left(50,[\mathrm{M}-1]^{+}\right)$, $526\left(100,[\mathrm{M}+1]^{+}\right), 527\left(28,[\mathrm{M}+2]^{+}\right)$. Anal. calcd. for $\mathrm{C}_{28} \mathrm{H}_{22} \mathrm{FeSSe}$ (525.34): C 64.02, H 4.22, S 6.10; found: C 64.09, H 4.31, S 6.11.

\section{3-Ferrocenyl-2,2,3-triphenyl thiirane (3d)}


Yield: $240 \mathrm{mg}$ (51\%). Yellow solid. M.p.: $150.2-152.2{ }^{\circ} \mathrm{C} .{ }^{1} \mathrm{H}$ NMR: ' 3.61 (s, 1H, CH-Fc), 3.87 (s, 2H, CH-Fc), 4.02 (s, 6H, CH-Fc), 6.90-6.93 (m, 3H, $\mathrm{CH}_{\text {arom. }}$ ), 7.05-7.09 (m, 1H, $\mathrm{CH}_{\text {arom. }}$ ), 7.12-7.20 (m, 6H, $\mathrm{CH}_{\text {arom. }}$ ), 7.23-7.29 (m, 2H, $\mathrm{CH}_{\text {arom. }}$ ), 7.38 (brs, $2 \mathrm{H}, \mathrm{CH}_{\text {arom. }}$ ) ppm. ${ }^{13} \mathrm{C} \mathrm{NMR:} \mathrm{'} \mathrm{64.8,} 69.6$ (2C, Cq), 67.8, 68.2, 68.9, 70.0, 71.2 (9C, CH-Fc), 91.8 (1C, C-Fc), 126.2, 126.6, 126.7, 126.8, 127.0, 127.1, 129.2, 131.5, 131.7 (15C, $\left.\mathrm{CH}_{\text {arom. }}\right), 138.9,140.1,140.9$ (3C, $\left.\mathrm{C}_{\text {arom. }}\right)$ ppm. IR: $\AA 3081(\mathrm{w}), 3052$ (m), 3025 (m), 2923 (w), 2850 (w), 1597 (w), 1491 (m), 1444 (m), 1107 (m), 1078 (m), $1028(\mathrm{~m}), 1002$ (m), $816(\mathrm{~m}), 780(\mathrm{~m}), 751$ (m), $723(\mathrm{~m}), 698(\mathrm{~s}), 660(\mathrm{~m}), 601(\mathrm{~m}), 512$ (m), $484(\mathrm{~m}) \mathrm{cm}^{-1}$. ESI-MS $439\left(24,[\mathrm{M}-\mathrm{S}+1]^{+}\right), 472\left(100,[\mathrm{M}]^{+}\right), 473\left(55,[\mathrm{M}+1]^{+}\right)$. Anal. calcd. for $\mathrm{C}_{30} \mathrm{H}_{24} \mathrm{FeS}$ (472.42): C 76.27, H 5.12, S 6.79; found: C 76.26, H 4.97, S 7.18 .

\section{ACKNOWLEDGMENTS}

The authors thank the National Science Center (PL-Cracow) for financial support within the project Maestro (Grant Maestro-3; Dec-2012/06/A/ST5/00219).

\section{REFERENCES}

1. (a) Jesberger, M.; Davis, T. P.; Barner, L. Synthesis 2003, 1929-1958; (b) Ozturk, T.; Ertas, E.; Mert, O. Chem. Rev. 2007, 107, 5210-5278.

2. Huisgen, R.; Li, X.; Giera, H.; Langhals, E. Helv. Chim. Acta 2001, 84, 981999.

3. Rohr, U.; Schatz, J.; Sauer, J. Eur. J. Org. Chem. 1998, 2875-2883.

4. (a) Barton, D. H. R.; Guziec, Jr., F. S.; Shahak, I. J. Chem. Soc., Perkin Trans. 1 1974, 1794-1799; (b) Guziec, F. S.; Sanfilippo, L. J. Tetrahedron 1988, 44, 6241-6285.

5. (a) Stepnicka, P. J., Ed., Ferrocenes: Ligands, Materials and Biomolecules, Wiley \& Sons Ltd., Chichester, U. K., 2008; (b) Hamera, R. Synlett 2015, in press.

6. Kowalski, K.; Karpowicz, R.; MlostoD, G.; Miesel, D.; Hildebrandt, A.; Lang, H.; Czerwieniec, R.; Therrien, B. Dalton Trans. 2015, 44, 6268-6276.

7. Kursanov, D.N.; Setkina, V. N.; Dolgova, S. P. Russ. Chem. Bull. 1979, 28, 814-816.

8. Sato, M.; Asai, M. J. Organomet. Chem. 1992, 430, 105-110. 
9. MlostoD, G.; Urbaniak, K.; G bicki, K.; Grzelak, P.; Heimgartner, H. Heteroatom Chem. 2014, 25, 548-555.

10. Pla| uk, D.; Zakrzewski, J. J. Organometal. Chem. 2009, 694, 1802-1806.

11. (a) Huisgen, R.; Kalwinsch, I.; Li, X.; MlostoD, G.; Eur. J. Org. Chem. 2000, 1685-1694; (b) Huisgen, R.; Li, X.; MlostoD, G.; Fulka, C. Eur. J. Org. Chem. 2000, 1695-1702.

12. MlostoD, G.; Urbaniak, K.; Linden, A.; Heimgartner, H. Helv. Chim. Acta 2015, $98,453-461$. 